\title{
Self-powered Water Meter for Direct Feedback
}

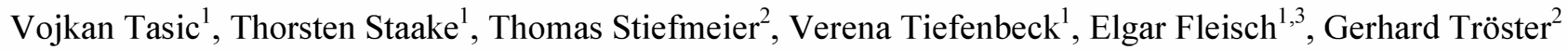 \\ ${ }^{I}$ Department of Management, Technology and Economics \\ ${ }^{2}$ Wearable Computing Lab \\ ETH Zurich \\ Zurich, Switzerland \\ \{vtasic, tstaake,vtiefenbeck\}@ethz.ch \\ ${ }^{3}$ Institute of Technology Management \\ University of St. Gallen \\ St.Gallen, Switzerland \\ elgar.fleisch@unisg.ch
}

\{stiefmeier,troester\}@ife.ee.ethz.ch

\begin{abstract}
Hot water usage accounts for $16 \%$ of household demand for energy, much more than lighting and cooking $(5 \%$ each) and is comparable to electricity usage for appliances $(21 \%)$. As a means of helping consumers to save hot water, we present a novel self-powered water consumption sensor that enables direct consumption feedback. We equipped 91 Swiss households with the sensors and recorded 3,164 individual showers during the period of three months. The presence of feedback during a shower resulted in the reduction of average shower water consumption from 79 I to 61 I $(-22.2 \%)$ per day and household. In addition to savings attributable to already installed flow restrictors, an average household could conserve 6,400 I of drinking water and $210 \mathrm{kWh}$ of heat energy (projected to one year). Furthermore, we show that the effects of direct feedback on water consumption did not decline over the course of the study.
\end{abstract}

\section{INTRODUCTION}

Recent scientific and commercial research has led to the development of cleaner ways of generating electricity and evermore energy-efficient devices. In addition to the advances in technology, high hopes are placed on behavioral energy conservation campaigns that aim at motivating and supporting individuals to act in an environmentally sustainable way [1][2][3]. Given the high importance of consumer behavior for sustainable energy usage, it is not surprising that such interventions have received considerable attention from researchers, companies, and policy makers [4].

In this context, special attention is paid to direct feedback interventions that inform individuals in a timely way about their energy usage or related environmental consequences following their behavior [5]. Theory often refers to this group of feedback types as: immediate, continuous or even real-time feedback. Researchers expect such information to serve as an enabler to better identify saving opportunities, and - given the bounded rationality of consumer choice - as a means to foster sustainable practices if combined with monetary (e.g. bonus points, rewards, etc.) and non-monetary (e.g. activation of social norms, goal setting, etc.) incentives.

A basis for direct feedback is Smart Metering, a system that serves as a prominent means to retrieve consumption information. Smart Metering relies on electronic measurement, storage, and communication of consumption data for electricity, gas, or water. In combination with suitable user interfaces (e.g., In-Home Displays, Smart Phone Apps), Smart Metering technology enables more timely feedback to endusers compared to conventional metering systems, which offer information on previous resource usage only at monthly or even yearly intervals.

Numerous studies deal with feedback effects on electricity consumption (see [6], [7] for a comprehensive overview). The studies report savings of $5 \%$ to $15 \%$, with a study duration being negatively correlated to the reported effects. The savings are achieved in settings where feedback is either not provided in a timely way or needs to be requested by the user (e.g. by logging in to a web portal). Critics of consumption feedback claim that the effects of feedback intervention decay over time. On the other hand, proponents of feedback technologies state that feedback interventions, if designed in a proper way, can turn desired behaviors into a habit that is stable over time.

In this paper, we contribute to the ongoing discussion in the field as we investigate effect size and its development over time for hot water usage. The feedback is provided immediately during resource usage and directly at the point of use. We have chosen feedback on hot water usage as a target measure, because it deserves special attention for several reasons: First, hot water consumption results in a large energy usage and $\mathrm{CO}_{2}$ emissions. About $5.7 \%$ to $6.9 \%$ of total $\mathrm{CO}_{2}$ emissions - including industry, transportation, and agriculture - result from individuals' water usage (see Figure 1.) [8]. Water heating itself takes $16 \%$ of total energy demand of an average household, which is comparable to the total energy consumption of electrical appliances (21\%) [4]. Second, there are only a limited number of points where hot water is withdrawn in a household, which enables easier direct feedback implementation. The main hot water extraction points are the shower and the bath, which account for around $42 \%$ of the hot water consumption in typical households [9]. Third, users have high factual and perceived control over water usage, since they can turn a shower on or off at any moment - unlike electricity demand, which is largely determined by a base consumption. Finally, water consumption feedback is easy to understand and users can relate to a specific amount of water as it can be materialized, which is not the case with electricity. Thus, it appears reasonable to expect strong effects of direct feedback on hot water usage. 


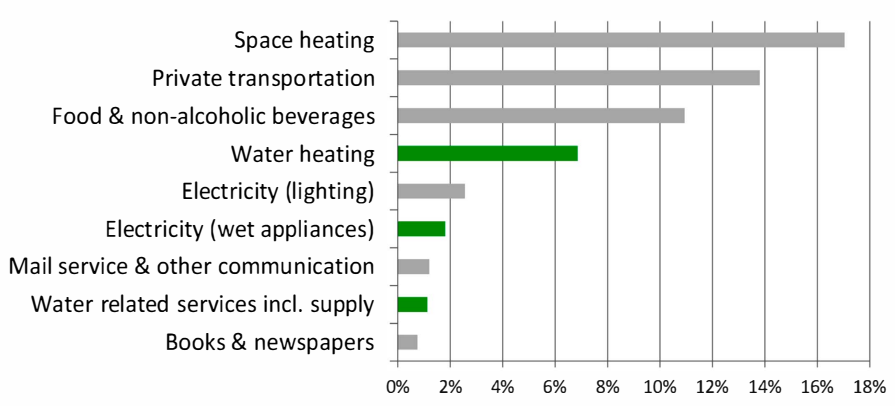

Figure 1. $\mathrm{CO} 2$ emissions by usage account [8]

To investigate the effects of direct feedback and trends over time, we developed a novel wireless sensor and display for water consumption measurement in the shower. Two hundred measurement/feedback devices were produced and handed out to Swiss households. In total, 3,164 shower sessions were logged with about 160 individual users. This paper reports saving effects of direct feedback during the study, which lasted for three months. With the presence of direct feedback, users significantly reduced their average water consumption for showering per day and per household (-22\%). Likewise, heat energy usage for hot water also declined by $0.6 \mathrm{kWh} /$ day per household. Interestingly, the effects of direct feedback remained stable over the course of study.

The next section summarizes published research on two topics which are essential for this paper: Firstly, research on feedback interventions which focus on direct feedback for energy consumption. Secondly, studies and projects with feedback methods and tools for water consumption/ measurement. Section 3 explains the study design and tools we developed for data collection. Section 4 presents user acceptance and participation during the study. In the fifth section, key findings are shown. Finally, section 6 concludes and outlines the implications for further research and development.

\section{RELATED WORK}

Despite the fact that consumer behavior and consumption feedback interventions are widely present in the literature [4], related work on direct feedback is sparse. This is mainly due to the lack of appropriate tools and methods for implementing direct feedback. Nevertheless, the first part of this section reviews a few important studies which involve direct feedback as a means of incentivizing sustainable energy behavior. The second part summarizes studies that deal with feedback on water consumption in particular, because water usage analysis is identified as, possibly, the most appropriate way to investigate effect size and stability properties of direct feedback.

\section{A. Direct feedback studies}

One of the first studies on feedback interventions were conducted in the 1970s, primarily in the domain of psychology. These studies were designed to provide feedback on electricity usage in households. Some consumers were provided with continuous real-time feedback, but most studies were based on monthly or weekly feedback. For instance, the study by Seligman and Darley [10] provided the first tangible results for electricity consumption feedback. Their study lasted one month; all participants were informed that air conditioners were the largest electricity consumers. Subsequently, half of the participating households received feedback, four times a week, on their electricity consumption. Households which received the feedback used $10.5 \%$ less electrical energy. The authors did not perform after-study measurements to investigate the decay of feedback effects.

Following the pioneering work in the late 1970s, numerous studies provided evidence for the effectiveness of direct feedback [5], [6]. The "information-deficit" model (see Figure 2 ) can be used for explaining direct feedback effects. It argues that direct feedback fills an "information vacuum" with additional insights [11]. Wilhite and Ling explained this model in [12]: They added additional consumption information to monthly bills, which incentivized consumers to use less energy.

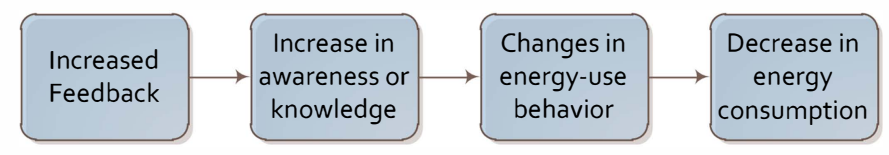

Figure 2. "Information-deficit" model [12]

Most studies have shown the impact of consumption feedback for electricity and gas. In particular, Darby et al. [6] states that direct feedback is extremely valuable and provides improved savings for electricity and gas in the region of 5 to $15 \%$. The first study including direct feedback was conducted in 1980 by McClelland and Cook [13]. They used home electricity monitors in order to provide direct electricity feedback to test households for the period of 11 months. Their study resulted in electricity savings of $12 \%$, compared to the households without electricity monitors installed.

The study on gas consumption feedback by Van Houwelingen and Van Raaij [14] compared direct feedback to feedback on a monthly basis. Households which received direct feedback saved $12.3 \%$ of gas, while the ones with monthly feedback saved only $7.7 \%$. This experiment showed the clear advantage of direct feedback over monthly consumption feedback.

As outlined previously, a number of studies dealing with direct feedback show that saving effects are strongly affected by the way feedback is presented. Moreover, reviewed studies based on direct feedback intervention achieved considerable saving effects, even up to $15 \%$ in certain cases [6]. Finally, direct feedback in these studies was usually implemented for gas or electricity consumption. Only a few studies investigated direct feedback on water consumption; these are reviewed in the next part.

\section{B. Studies on water consumption feedback}

One of the first studies on water usage in the shower was carried out by Kappel and Grechenig in 2009 [15]. They employed a non-conventional approach to deliver direct feedback. Instead of standard displays, they used ambient displays consisting of 16 diodes representing fictive water levels based on shower usage. Their study lasted for three weeks and included four households. This experiment resulted in the average saving of 10 liters per shower session. 
Another experiment on water usage which included direct feedback, was authored by a group of Australian researchers and engineers led by Willis et al. [16]. The team investigated the effects of a shower monitor deployed in 44 households in the Melbourne region. During the first month of the study, the devices measured the baseline consumption (measurement without display) and thereafter automatically switched into the feedback mode to display current water consumption. In the feedback mode, the devices also provided an acoustic alarm signal when a user-adjustable water volume was exceeded. Four additional devices with deactivated displays measured consumption over the entire six months to adjust for seasonal fluctuations. Average savings of $15 \%$ were reported for feedback mode compared to baseline consumption. The study offers additional insights into the effects of real-time feedback for water usage. It is, however, difficult to apply the findings to countries where water is abundant. The effects seen in Australia might be stronger than in Europe due to a more pronounced problem awareness, or they might be weaker because a considerable portion of the savings potential has already been realized. Another drawback of the study is the intrusiveness of the equipment installation, possibly increasing participants' feelings of being in an experimental situation and fostering socially desired behavior.

In contrast to Willis et al., IBM conducted a pilot project with conventional Smart Water Meters [17]. The meters were connected to 303 main water supplies of households in the city of Dubuque, Iowa, USA. In this project, 151 households were granted access to an online portal that provided information on their water usage, based on hourly aggregated data, along with information on trends and a personal ranking. Additional 152 households served as control group and received no feedback information. The treatment group saved $6.6 \%$ more than the control group over the period of nine weeks. However, the study cannot be considered as a direct feedback study, since it provided only hourly data. Nevertheless, it is important as the first domestic water consumption study with a larger number of participants and important for comparison with our direct feedback study.

\section{METHODS}

\section{A. Technology/infrastructure}

In cooperation with Swiss company, we developed and manufactured measurement and display units. It consists of two main parts: a self-powered measurement device (a cylindrical sensor with diameter of $14.7 \mathrm{~mm}$ and height of $51 \mathrm{~mm}$, see Figure 3.1 and Figure 4) and a bright, waterproof, display module that is battery-powered and can display four sevensegment digits. It communicates with the measurement module via an infrared protocol (see Figure 3). The display automatically becomes active when the water fixture is turned on. Thus, no user action is required during the experiment. After a longer period of inactivity, the display automatically resets and reinitializes the measurement routine. Moreover, the device automatically aggregates consecutive extractions as one shower session if they follow each other within 3 minutes (e.g.: extraction from 0.001 to $34.05 \mathrm{l}$; pause to wash hair; measuring from 34.051 to $68.28 \mathrm{l}$; reset to 0.001 after three minutes of inactivity) and stores the aggregated data of each extraction event.

The display unit operation and handling is simplified: when the sensor enters the active mode (shower session starts), the sensor unit transmits the data via infrared to the display unit (one-way communication). When the communication is initialized, the display unit logic turns four seven-segment displays on and starts to receive data from the sensor.

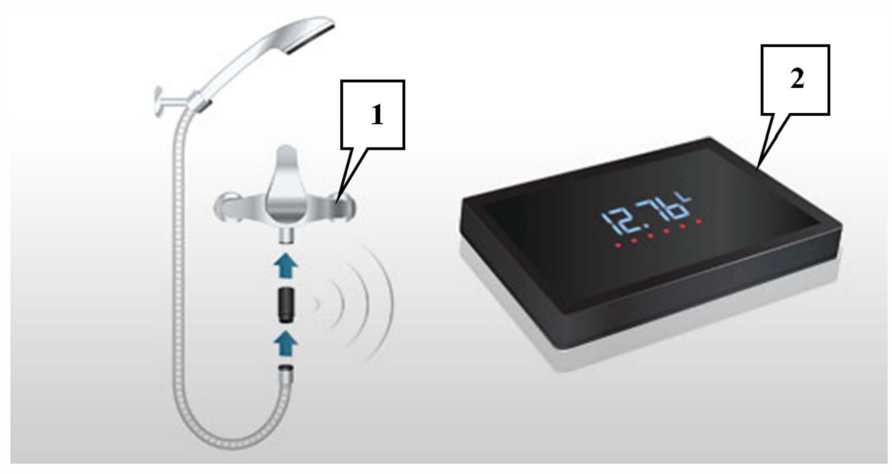

Figure 3. Installation of the Smart Water Meter; 1 - Sensor, 2 display/receiver

The sensor unit comprises two novel solutions to overcome major shortcomings of existing products and prototypes: First, it allows tool-free installation - as illustrated on Figure 3, it is very easy to mount the sensor in the shower and it takes only two minutes of work. This approach solves the problem of complex mounting, which usually requires a plumber. Second, the self-powered sensor removes the need for a battery or other external power supply. Our sensors have a small internal power generator (turbine), which harvests kinetic energy from the water flow, making it self-powered (see Figure 4). Harvested energy (in form of electrical energy) is used to power the microcontroller (MCU) and the communication electronics.

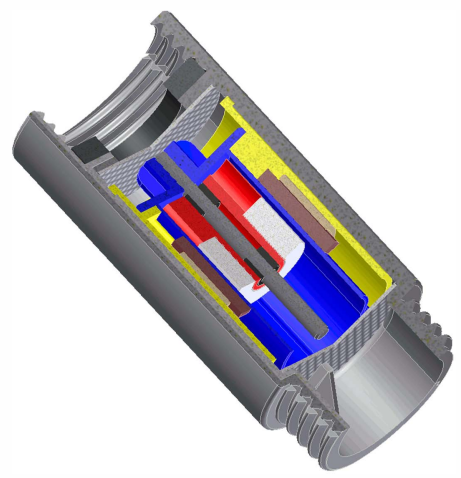

Figure 4. Cross-section of measurement unit (sensor): gray - unit housing, blue - turbine, yellow - sensor housing

Specifications of the sensor unit:

- Start-up flow: The generator starts to produce electricity when the water flow reaches 5 liters/min.

- $\quad$ Pressure drop: Flow at the pressure drop of 3 bar is 15 liters/min. 
- Dimensions: Cylindrical shape with diameter of $14.7 \mathrm{~mm}$ and height of $51 \mathrm{~mm}$.

- Communication: Infrared technology with $5 \mathrm{~m}$ range.

- Frequency/Flow: Rotational frequency of the generator: $270 \mathrm{~Hz} @ 9$ liters/min (water flow).

Main functions of the sensor unit:

- Electricity generation: Electricity is generated in a power generator that transforms kinetic energy from the water flow into electrical energy (see Figure 4).

- Rectification: Since the generator is AC, we developed a simple AC/DC rectifier for powering the embedded electronics.

- Water flow-rate measurement: Flow rate and water volume calculation is based on a generator frequency and implemented using a look-up table, as the relationship between generator frequency and water flow is non-linear.

- Temperature measurement: The sensor unit also measures and logs water temperature to calculate heat energy (in hot water).

- Wireless communication: Measurements are locally stored and processed in the MCU, then send to the display unit via infrared communication protocol.

The display unit uses sensor measurements to provide direct feedback to users on their water consumption for showering (see Figure 3.1 and Figure 5). Raw data reception from the sensor is implemented with one infrared sensor (see Figure 5.6) which is controlled by the MCU (see Figure 5.3) in the display unit. After the data are received, they are processed for displaying. The displays are able to show volume of water consumed, water temperature or energy spent for hot water.

The display is powered by four AAA batteries which had sufficient capacity for typical usage during the study of three months. Since the communication is designed in a way that there is no need for pairing, the display is "listening" for the infrared signal the whole time (even when the sensor is off). This approach significantly reduces the complexity of the firmware design at the cost of reducing battery life.

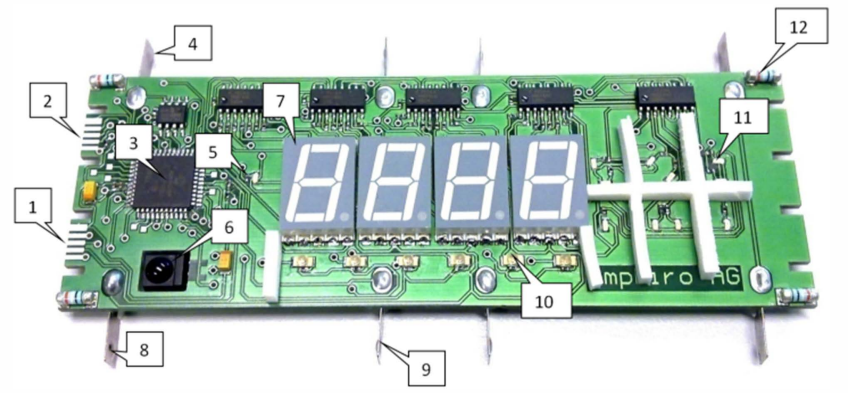

Figure 5. Electronics of the display unit; Legend: 1 - Programming socket, 2 Readout socket, 3 - PIC microcontroller, 4 - 0 V contact, GND, 5 - Minus sign (-) LED (blue), 6 - IR receiver, 7 - seven-segment LED displays, 8 - 6V contact (VLED), $9-4.5 \mathrm{~V}$ contact $\left(\mathrm{V}_{\mathrm{Log}}\right), 10-\mathrm{LED}$ bar, red, $11-\mathrm{Symbol}$ LEDs, blue, 12 - Spacer MELF

\section{B. Study design}

This research study is based on a field experiment in Switzerland that was conducted between September 2010 and January 2011. For the recruitment of participants, 200 Smart Water Meters were distributed to Swiss households. Participation in the study was optional; participants were informed that individual user and questionnaire data would not be disclosed and remain anonymous.

Before using the Smart Water Meters, participants had to fill out the first questionnaire. Participants were asked to return the devices immediately if they were unwilling to participate in the study. In addition to demographics, we collected data on environmental attitudes and technology affinity. A follow-up questionnaire was distributed right after the experiment contained enough data for an analysis, which was usually after the period of three months.

The Smart Water Meters were built to comply with Switzerland's prevalent shower design (a hand shower with a flexible hose connected to a mixer tap) and allowed for easy installation between the shower faucet and the shower hose. The four-step installation was described in a manual: "remove the hose from the faucet - attach the Smart Meter (the black cylinder) to the faucet - attach the hose to the Smart Meter place the waterproof monitor within a sight in your bathroom." Step-by-step illustrations visualized the easy installation process. The system required no configuration or pairing of sensor with display module (receiver). To provide help during installation, we set up the hotline number in case participants had questions, but there were no requests for telephone support.

We designed the experiment in a way that during the first nine shower sessions, the devices did not provide any feedback on water consumption. Instead, it showed only the number of showers until the display started to show information on water usage. This phase of the experiment is used to measure the baseline consumption. After the baseline measurement part of the experiment, the devices showed aggregated water volume consumed during one shower in real-time fashion.

\section{Response RATE AND USER AcCEPTANCE}

Ninety-one devices, out of two-hundred distributed, were installed and used; 95 were returned unused. Fourteen devices were not returned until the completion of the study. Sixty-one devices, out of 91 installed, contained usable records with more than 24 measurements, and 44 of those obtained records with more than 40 measurements. On average, the devices stored 52 measurements (nine baseline mode plus 43 in feedback mode). In total, 3,164 shower sessions were recorded in 61 households with about 160 individual users.

Thirty devices, out of 91 installed, contained useless data. There are several reasons for this high number of bad measurements: too few showers taken, obvious manipulation of the experiment (e.g., the shower was operated nine times on the first day to avoid the baseline measurement phase to immediately enter feedback mode), or hardware failure. 
The total participation rate was $49 \%$ (91 devices used, 95 devices not used) (see Figure 6). There are different possible reasons for not using the study devices:

- Concerns about data privacy

- Lack of interest

- Unwillingness to consider consumption while showering

- Difficulties during installation (e.g., the shower hose could not be screwed off)

- Incompatible screw threads at some bathroom installations (selected showers use $3 / 8$ " instead of $1 / 2$ " windings)

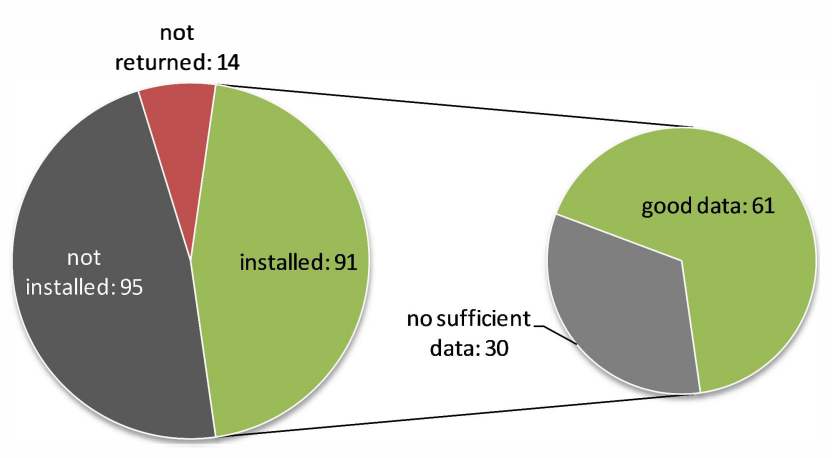

Figure 6. Participation and share of usable records

Participants completed 93 initial questionnaires and 100 follow-up questionnaires at the end of the study. In total, forty households returned both questionnaires and produced valid measurements. Overall, the number of used devices and successfully completed questionnaires was within the expected range and was taken into account when deciding upon the number of distributed devices.

\section{IMPACT ON CONSUMPTION}

The effects we report here are based on measurements of 61 devices, which recorded, 3,164 shower sessions (in 61 households with about 160 individual users). Changes in water usage are given either as absolute or relative change between the baseline measurement (without direct feedback influence) and direct feedback mode (the display shows the water volume per shower session).

With direct feedback, users reduced their average shower water consumption per day and household from 79 l to 611 ($22.2 \%$ ). Similarly, heat energy usage for showering using hot water declined by $0.6 \mathrm{kWh}$ per day and household. Projected to one year, in addition to savings attributed to already present flow restrictors, an average household could save 6,400 l of drinking water and $210 \mathrm{kWh}$ of energy with presence of direct feedback in the shower.

The drop in water volume spent for showering is due to the shortening of individual shower sessions. Although participants reduced the duration of individual showers, we found no impact on shower frequency. Besides, we also found no changes in shower temperature; the latter, however, might be an artifact from the low resolution of one degree Celsius of our devices. Additionally, participants tended to keep the flow rates stable during the course of study. As an exception, the data from three households showed a sharp and persistent drop of the flow rate within the feedback period, which might indicate the replacement of a conventional shower head by a low-flow model.

The savings effects varied considerably between different households. Sixty-two percent of the households reduced their water and energy consumption per shower by at least $10 \%$, and $28 \%$ achieved saving rates of more than $30 \%$. However, $20 \%$ of the households did not save a significant amount of water and energy, and $18 \%$ consumed more when feedback information was present (see Figure 7).

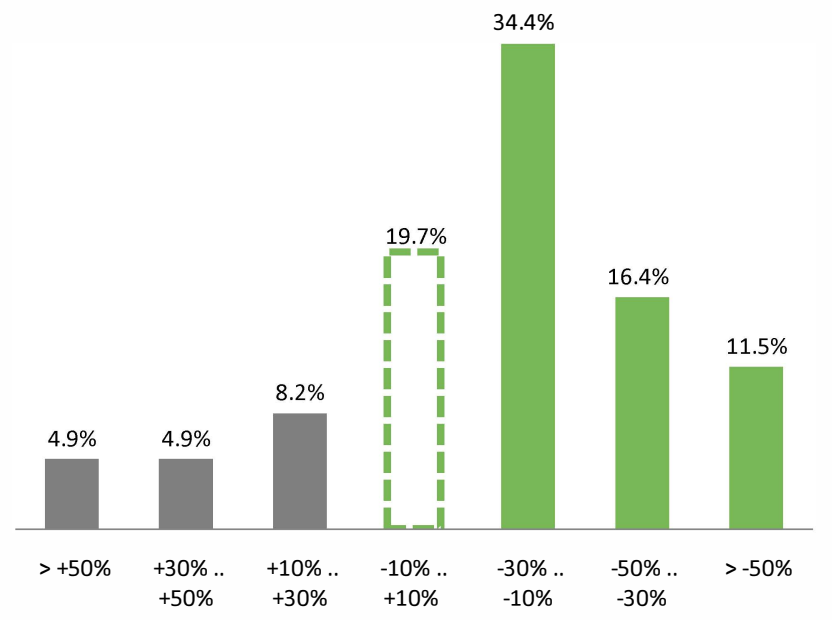

Figure 7. Distribution of the changes in daily water and energy usage per household $\left(\mathrm{N} \_\right.$blind $=548$, N_treat $=2616$ )

For the further analysis, we report savings effects in percent of the baseline measurement. Please note that the average savings expressed relatively to the individual baseline do not necessarily match the overall savings in water or energy consumption. Absolute savings in water and energy consumption (the relevant measure from an environmental perspective) in the study are $22.2 \%$, whereas the average savings per household over baseline are $12.9 \%$. The latter number is smaller, as it is influenced disproportionally by users with low average consumption who increase their water demand slightly with respect to the absolute value, but strongly when expressed as the share of baseline consumption.

A more detailed analysis revealed the dependency of savings effects on baseline consumption. Although virtually all users with high baseline consumption decreased their demand; consumers who used very little water per shower initially were prone to increase water usage thereafter. This "constructive and destructive" effect of descriptive feedback is well-known and discussed in [18], where Schultz et al. showed that feedback can even lead to an overuse of resources among already efficient consumers [18]. When looking at average savings per household over baseline after dividing households into aboveand below-median consumers, the effects of baseline consumption on savings effects become apparent (see Figure $8)$. Above-median users saved significantly more (20.2\%) than below-median users. Below-median users saved in average 
$4.9 \%$. The reason for not adding up to reported $22.2 \%$ of savings can be explained by the following scenario: Participant $\mathrm{A}$ increased his consumption from $20 \mathrm{l} /$ day to $30 \mathrm{l} /$ day $(+50 \%)$ and participant $\mathrm{B}$ reduced his consumption from $100 \mathrm{l} /$ day to 70 1/day $(-30 \%)$. While an average water consumption increased by $10 \%$ with direct feedback, the overall consumption is reduced by $17 \%$ (from 120 to 100 liters).

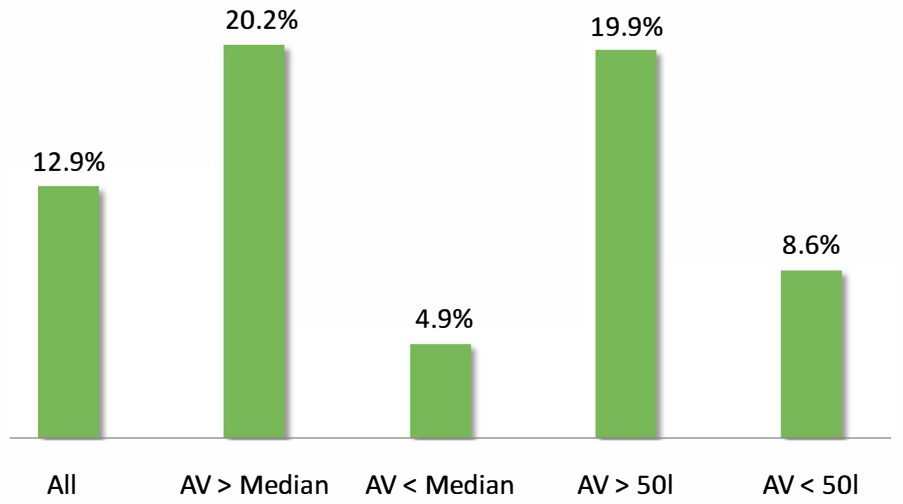

Figure 8. Savings effects by baseline consumption (AV) (N_all=3164, $\mathrm{N}_{-}>\mathrm{M}=1685, \mathrm{~N}<\mathrm{M}=1479, \mathrm{~N}>50$ liters$=1094, \mathrm{~N}_{-}<50$ liters $=2070$ )

The practical importance of the findings depends heavily on the long-term stability of the savings effects. In order to measure the stability of the effects across the duration of the study, we split the feedback period into two phases of equal length (1,208 and 1,207 measurements). Although the first half of the feedback period showed savings of $24 \%$, we can report savings of $20 \%$ during the second half (see Figure 9). Therefore, the effect of feedback on water consumption did not decline considerably over the course of the study.

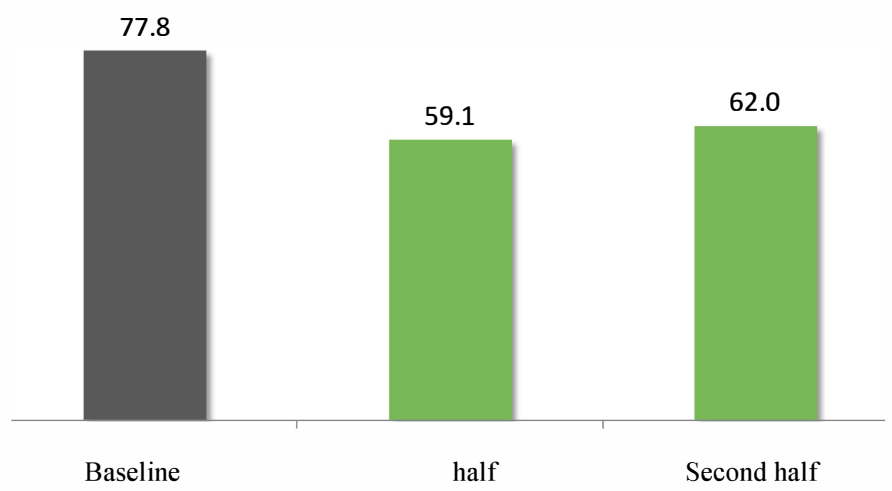

Figure 9. Average water consumption per day for first and second half of the treatment in comparison to baseline (in liter; N_blind $=31$ N_H1 $=1208$, N_H2=1207)

\section{DISCUSSION/CONCLUSION}

Pursuing the idea that direct feedback is more efficient than other methods [6], we developed a technology for providing direct feedback in the shower. This paper presents the novel technology and the study we conducted in order to explore direct feedback effects.
Our smart water meter consists of two parts: the selfpowered sensor with embedded infrared communication and the display module which receives the data from the sensor, adapts it, and provides it to a user. Our novel approach turns regular faucets into smart objects with the ability to communicate to direct feedback "providers" (in-shower displays, in-home displays, web portals, smart phones, etc.). Furthermore, our technology enables one more step towards including water related appliances to the IOT domain.

In order to test our device and its direct feedback feature, we conducted the study with around 200 Swiss households over three months. Response / participation rate was in line with the expectations (49\%). Some of the reasons for not participating in the study are: concerns about data privacy, lack of interest, unwillingness to consider consumption while showering, difficulties with installation. After the study, we collected the data from 91 households with 160 individual consumers and 3,164 individual shower sessions. The dataset fulfilled quantitative and qualitative requirements for investigating effect size and stability of the direct feedback.

The saving results are higher than expected. Absolute savings, which are relevant from an environmental perspective, are $22.2 \%$, while the average savings per household over baseline are $12.9 \%$. Consumers with a high baseline consumption realized much higher savings than users with a low baseline consumption (this is clearly depicted in Figure 8). Also, the savings effects remained stable during the course of study, with very slight tendency to decay (Figure 9). Comparing to similar field studies for water consumption with direct feedback, our experiment setup achieved the highest savings in water volume per day and household (18 liters). As a consequence, each household saved on average per day 0.6 $\mathrm{kWh}$ of heat energy. Projected to one year, the average household could conserve 6,400 liters of drinking water and $210 \mathrm{kWh}$ of heat energy. In a fictive scenario where each European household would have such a smart water meter with direct feedback, the energy savings would add up to around $42 \mathrm{TWh}$ (projected to one year). This amount of saved energy equals to the yearly production of ten modern coal power plants $(600 \mathrm{MW})$ which would emit 33 millions of tons of $\mathrm{CO}_{2}$. The construction of such plants alone would cost 4.7billion USD. If this amount of energy is to be generated with solar panels within one year, it would require $3.3 \mathrm{GW}$ of installed power and occupy around 3000 acres of land (surface of 1700 soccer fields). Savings in drinking water, projected to one year, would also be enormous. Europe would save $1.3 \mathrm{~km}^{3}$ of drinking water which cost 130 million USD to produce.

The future development of the device (smart water meter) will include two important milestones: First, the display of future smart water meters will be also powered by the microgenerator, which completely removes the need for batteries. Second, wireless connectivity will be added and it will be based on RF (Radio Frequency) communication. The RF connectivity feature will add additional value to water related appliances because it will be able to harness the power of social networks. 


\section{REFERENCES}

[1] T. Dietz, G. T. Gardner, J. Gilligan, P. C. Stern, and M. P. Vandenbergh, "Household actions can provide a behavioral wedge to rapidly reduce US carbon emissions," Proceedings of the National Academy of Sciences, vol. 106, no. 44, pp. 18452-18456, Nov. 2009.

[2] D. McKenzie-Mohr, L. S. Nemiroff, L. Beers, and S. Desmarais, "Determinants of Responsible Environmental Behavior," Journal of Social Issues, vol. 51, no. 4, pp. 139-156, Jan. 1995.

[3] L. Steg and C. Vlek, "Encouraging pro-environmental behaviour: An integrative review and research agenda," Journal of Environmental Psychology, vol. 29, no. 3, pp. 309-317, Sep. 2009.

[4] O. International Energy Agency, "Worldwide Trends in Energy Use and Efficiency - Key Insights from IEA Indicator Analysis." 2008.

[5] S. Geller, "The challenge of increasing proenvironmental behavior," in Handbook of environmental psychology, John Wiley and Sons, 2002, pp. 525-540.

[6] S. Darby, "The effectiveness of feedback on energy consumption," $A$ review for DEFRA of the literature on metering, billing and direct displays, p. 21, 2006.

[7] W. Abrahamse, L. Steg, C. Vlek, and T. Rothengatter, "A review of intervention studies aimed at household energy conservation," Journal of Environmental Psychology, vol. 25, no. 3, pp. 273-291, 2005.

[8] T. Jackson, E. Papathanasopoulou, P. Bradley, and A. Druckman, "Analysing the Role of Lifestyles in Determining UK Household Energy Demand and GHG Emissions: Predictions and Scenarios to 2050," in Conference on Energy Economics and Technology. Long-term Energy Trends - Where will we be in 2050, 2009.

[9] C. Aguilar, D. White, and D. L. Ryan, "Domestic water heating and water heater energy consumption in Canada," Canadian Building Energy End-Use Data and Analysis Centre, 2005.

[10] C. Seligman and J. M. Darley, "Feedback as a means of decreasing residential energy consumption," Journal of Applied Psychology, vol. 62 , no. 4 , pp. 363-368, 1977.
[11] T. Hargreaves, M. Nye, and J. Burgess, "Making energy visible: A qualitative field study of how householders interact with feedback from smart energy monitors," Energy Policy, vol. 38, no. 10, pp. 6111-6119, 2010.

[12] H. Wilhite and R. Ling, "Measured energy savings from a more informative energy bill," Energy and Buildings, vol. 22, no. 2, pp. 145$155,1995$.

[13] L. McClelland and S. W. Cook, "Energy conservation effects of continuous in-home feedback in all-electric homes," Journal of Environmental Systems, vol. 9, no. 2, pp. 169-173, 1979.

[14] J. H. van Houwelingen and W. F. van Raaij, "The Effect of GoalSetting and Daily Electronic Feedback on In-Home Energy Use," Journal of Consumer Research, vol. 16, no. 1, pp. 98-105, Jun. 1989.

[15] K. Kappel and T. Grechenig, "show-me': water consumption at a glance to promote water conservation in the shower," in Proceedings of the 4th International Conference on Persuasive Technology, 2009, p. 26.

[16] R. M. Willis, R. A. Stewart, K. Panuwatwanich, S. Jones, and A. Kyriakides, "Alarming visual display monitors affecting shower end use water and energy conservation in Australian residential households," Resources, Conservation and Recycling, vol. 54, no. 12, pp. 1117-1127, 2010.

[17] Milind Naphade, David Lyons, Chris A. Kohlmann, and Cindy Steinhauser, "IBM Research - Watson, Smart Water Pilot Study Report." 2011.

[18] P. W. Schultz, J. M. Nolan, R. B. Cialdini, N. J. Goldstein, and V. Griskevicius, "The Constructive, Destructive, and Reconstructive Power of Social Norms," Psychological Science, vol. 18, no. 5, pp. $429-434$, May 2007. 